\title{
Correction to: Sensory signs and symptoms in women with self-reported breast cancer-related lymphedema: a case-control study
}

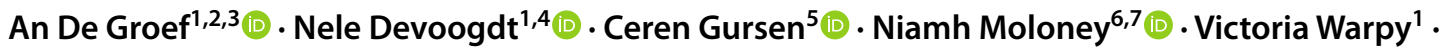 \\ Jolien Daelemans ${ }^{1} \cdot$ Lore Dams $^{1,2,3}$. Vincent Haenen ${ }^{1,2,3}$. Elien Van der Gucht ${ }^{1,2,3}$ - An-Kathleen Heroes ${ }^{1,4}$. \\ Tessa De Vrieze ${ }^{1,2}$ (E) Elizabeth Dylke ${ }^{8}$ (i)
}

Published online: 30 October 2021

(c) Springer Science+Business Media, LLC, part of Springer Nature 2021

\section{Correction to: Journal of Cancer Survivorship https://doi.org/10.1007/s11764-021-01100-z}

In this article the title was incorrectly given as 'Sensory signs and symptoms in women with self-reported breast cancer-related lymphedema: a case-control study close up' but should have been 'Sensory signs and symptoms in women with self-reported breast cancer-related lymphedema: a case-control study'.

The error in the abstract has been corrected.

The correct abstract is given below:

The original article can be found online at https://doi.org/10.1007/ s11764-021-01100-z.

An De Groef

an.degroef@kuleuven.be

https://caredon.org/about-us/team/prof-de-groef

1 Department of Rehabilitation Sciences, KU Leuven University of Leuven, Leuven, Belgium

2 Department of Rehabilitation Sciences and Physiotherapy, University of Antwerp, Antwerp, Belgium

3 Pain in Motion International Research Group, Brussel, Belgium

4 Department of Vascular Surgery and Department of Physical Medicine and Rehabilitation, Centre for Lymphedema, University Hospitals Leuven, Leuven, Belgium

5 Faculty of Physical Therapy and Rehabilitation, Hacettepe University, Ankara, Turkey

6 Department of Health Professions, Faculty of Medicine, Health and Human Sciences, Macquarie University, Sydney, NSW, Australia

7 THRIVE Physiotherapy, Channel Islands, Guernsey, UK

8 Faculty of Medicine and Health, The University of Sydney, Lidcombe, NSW, Australia

\section{Purpose}

It is not clear to what extent signs and symptoms other than arm swelling, including pain, altered sensory function, and body perception disturbances, differ between women with measurable and non-measurable breast cancer-related lymphedema (BCRL). A case-control study was performed to compare these signs and symptoms between (1) women with self-reported BCRL with objectively measurable swelling; (2) women with self-reported BCRL without objective confirmation; and (3) a control group with no self-reported BCRL.

\section{Methods}

The three groups were compared for (1) the severity of selfreported signs and symptoms of BCRL, (2) problems in functioning related to BCRL, (3) pain-related outcomes, (4) sensory functions, and (5) body perception.

\section{Results}

All self-reported outcomes related to signs and symptoms of BCRL and problems in functioning were significantly different between the control group and the other two groups with and without measurable self-reported BCRL ( $p<0.001-0.003)$. Except for "skin texture" $(p=0.01)$, "hand swelling" $(p=0.301)$ and "difficulty writing" $(p=0.676)$, no differences were found between groups. For pain-related outcomes, sensory function, and body perception, significant differences were found for the mechanical detection threshold $(\mathrm{p}<0.01)$ and self-reported disturbances in body perception $(\mathrm{p}<0.001)$ between the selfreported BCRL groups and control group. 


\section{Conclusions}

Diverse signs and symptoms related to BCRL, sensory function, and perception were different among women with self-reported BCRL compared to controls. No differences between women with and without measurable self-reported BCRL were found.

\section{Implications for Cancer Survivors}

The presence of self-reported BCRL, with or without measurable swelling, is a first indication for the need of further diagnostic evaluation.
Keywords: Breast neoplasms, Lymphedema, Signs and symptoms, Sensory function, Body perception, Pain

The original article has been updated.

Publisher's note Springer Nature remains neutral with regard to jurisdictional claims in published maps and institutional affiliations. 